\title{
PROTOCOLO DE SAMBA BRASILEIRO PARA REABILITAÇÃO CARDÍACA
}

THE BRASILIAN SAMBA PROTOCOL FOR CARDIAC REHABILITATION

PROTOCOLO DE SAMBA BRASILEÑO PARA REHABILITACIÓN CARDIACA

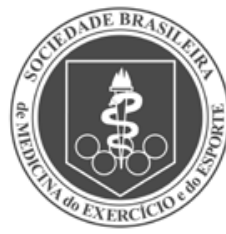

Artigo Original

Original ARTICLE

Artículo Original
Helena de Oliveira Braga (Acadêmica de Fisioterapia)

Ana Inês Gonzáles

(Fisioterapeuta)

Sabrina Weiss Sties ${ }^{1}$

(Fisioterapeuta)

Gabriela Maria Dutra de Carvalho' (Letróloga)

Almir Schmitt Netto

(Educador Físico)

Oswaldo Augusto Campos'

(Acadêmico de Fisioterapia)

Daiane Pereira Lima'

(Fisioterapeuta)

Tales de Carvalho

(Cardiologista)

1. Universidade do Estado de Santa Catarina (UDESC), Núcleo de Cardiologia e Medicina do Exercício (NCME), Florianópolis, SC, Brasil.

\section{Correspondência:}

Rua Pascual Simone, 358, Coqueiros. 88080-350. Florianópolis, SC, Brasil. helenadeoliveirabraga@gmail.com

\section{RESUMO}

Introdução: O samba é uma dança de movimentos intensos e variados que proporcionam alegria e boa resposta cronotrópica, sendo plausível a expectativa de que beneficie o sistema cardiovascular. Objetivo: Desenvolver e avaliar um protocolo de samba brasileiro visando o treinamento físico na reabilitação cardíaca. Métodos: Quinze indivíduos com doença arterial coronariana estável, idade 60,74 $\pm 5,96$ anos, realizaram teste cardiopulmonar máximo com determinação da frequência cardíaca $(\mathrm{FC})$ correspondente ao $\mathrm{VO}_{2 \text { pico }}$ e primeiro e segundo limiares ventilatórios. Durante seis sessões executaram vinte passos de samba, em três andamentos musicais, classificados por metrônomo em lento, médio ou rápido. Posteriormente, realizaram três sessões para verificar o comportamento da FC e a percepção de esforço. A análise descritiva foi utilizada na caracterização da amostra e verificação da $\mathrm{FC}$, o teste $t$ ou teste de Wilcoxon foram utilizados para comparar o comportamento da FC (nas diferentes sessões), com nível de significância de 5\%. Resultados: Houve boa adaptação aos passos de samba, com FC situando-se entre $62 \%$ e $72 \%$ da $\mathrm{FC}_{\text {pico. }}$. Durante as sessões de dança os pacientes mantiveram-se em $86 \pm 4,17 \%$ do tempo na zona alvo proposta para treinamento físico na reabilitação (60\% a $90 \%$ da $\mathrm{FC}_{\text {pico }}$ e $77 \pm 2,26 \%$ do tempo na zona alvo considerada ideal, com a $\mathrm{FC}$ situada entre o primeiro e o segundo limiares ventilatórios. Durante todo o tempo os pacientes referiram percepção de esforço de leve a moderada. Conclusão: Os pacientes demonstraram boa adaptação ao protocolo de samba, que se mostrou em condições de ser adotado como proposta de treinamento na reabilitação cardíaca.

Palavras-chave: exercício, terapia através da dança, doenças cardiovasculares.

\section{ABSTRACT}

Introduction: Samba requires intense and varied movements that provide happiness and good chronotropic response making plausible to expect its benefits on cardiovascular health. Objective: To develop and evaluate a brazilian samba protocol aiming at physical training in cardiac rehabilitation. Methods: Fifteen patients with stable coronary artery disease, aged 60.74 \pm 5.96 years, underwent maximal cardiopulmonary test with determination of heart rate (HR) corresponding to the peak $\mathrm{VO}_{2}$ and first and second ventilation thresholds. During six sessions, they underwent twenty samba steps, in three music tempo classified by a metronome (slow, medium and fast). Subsequently, they underwent three sessions to verify the HR behavior and perception of effort. Descriptive analysis has been utilized in order to characterize the sample and check HR; T test or Wilcoxon test have been used to compare HR behavior (in different sessions) with significance level of 5\%. Results: There was good adaptation to the samba steps, with HR between $62 \%$ and $72 \%$ of peak $H R$. During the dance sessions patients remained $86 \pm 4.17 \%$ of time in the target zone proposed for physical training in rehabilitation (60\% to $90 \%$ of HR peak) and $77 \pm 2.26 \%$ of time in the target zone considered ideal, with HR situated between the first and the second ventilation threshold. Throughout the time the patients reported having perceived mild to moderate effort. Conclusion: Patients showed good adaptation to samba protocol, which proved being able to be adopted as a proposal for training in cardiac rehabilitation.

Keywords: exercise, dance therapy, cardiovascular diseases.

\section{RESUMEN}

Introducción: Samba es una danza de movimientos intensos y variados que traen alegría y buena respuesta cronotrópica, y es plausible esperar que beneficia el sistema cardiovascular. Objetivo: Desarrollar y evaluar un protocolo de samba brasileño destinado a entrenamiento físico en la rehabilitación cardiaca. Métodos: Quince pacientes con enfermedad coronaria estable, edad 60,74 \pm 5,96 años, fueron sometidos a prueba de esfuerzo cardiopulmonar máximo para determinar la frecuencia cardíaca $(F C)$ correspondiente a $V_{2} O_{2 i c o}$ y primer y segundo umbrales ventilatorios. Durante seis sesiones realizaron veinte pasos de samba, en tres movimientos musicales clasificados en orden de metrónomo a lento, medio o rápido. Posteriormente se realizaron tres sesiones para comprobar el comportamiento de la FCy el esfuerzo percibido. Se realizó un análisis descriptivo para la caracterización de la muestra y la verificación de la FC; se utilizaron la prueba to prueba de Wilcoxon para comparar el comportamiento de la FC (en diferentes sesiones), con un nivel de significación del 5\%. Resultados: Hubo una buena adaptación a los pasos de samba, con la FC entre el $62 \%$ y el $72 \%$ de $F C_{\text {picco }}$. Durante las sesiones de baile, los pacientes se mantuvieron en $86 \pm 4,17 \%$ del tiempo en la zona de destino propuesta para el entrenamiento físico en rehabilitación (60\% a 90\% de $F C_{\text {picd }}$ y $77 \pm$ 
2,26\% del tiempo en la zona de destino considerado óptimo, con la FC situada entre el primero y el segundo umbral ventilatorio. Todo el tiempo la percepción percibida pelos pacientes fue de esfuerzos leves a moderados. Conclusión: Los pacientes mostraron una buena adaptación al protocolo de samba, que resultó en condiciones de ser adoptado como una propuesta para el entrenamiento en rehabilitación cardiaca.

Palabras clave: ejercicio, terapia a través de la danza, enfermedades cardiovasculares.

\section{INTRODUÇÃO}

O reconhecimento do exercício físico como recurso terapêutico para pacientes cardiopatas é consensual ${ }^{1-3}$, pois pode proporcionar melhora da angina em repouso, atenuação da gravidade da isquemia, ganho de aptidão física e controle de fatores de risco 1,3,4.

Os programas de reabilitação cardíaca reduzem a morbidade e a mortalidade de coronariopatas ${ }^{1,5}$. No entanto, existe dificuldade em manter o entusiasmo dos pacientes, visando à aderência ao tratamento ${ }^{6,7}$, tornando necessárias novas estratégias, o que tem motivado pesquisas no Brasil e no exterior ${ }^{8-10}$.

Nesse contexto, a dança configura-se como alternativa eficaz ${ }^{11,12}$, sendo uma atividade lúdica e prazerosa, capaz de proporcionar motivação para realização de atividades físicas ${ }^{13}$, beneficiar os aspectos social, emocional e cognitivo, comprovadamente melhorando a capacidade física e a qualidade de vida (QV) de indivíduos saudáveis ${ }^{14,15}$, já sendo utilizada na recuperação de pacientes com doenças crônicas não transmissíveis ${ }^{11,12,16-18}$. Estudos têm demonstrado que a dança pode proporcionar resultados similares ou até superiores no aprimoramento da aptidão física e das condições cardiovasculares, com destaque para a melhora da

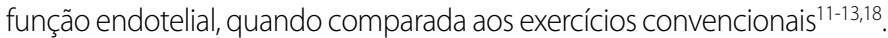

"A dança é uma atividade que pode ser desempenhada com músicas de variados gêneros e diferentes andamentos musicais, sabendo-se que a resposta cronotrópica de um indivíduo pode ser fortemente influenciada de acordo com o andamento musical utilizado"19,20. O samba apresenta-se de várias formas, é dançado individualmente ou em casal, tendo sido introduzido no estado da Bahia, Brasil por escravos africanos, posteriormente popularizado no estado do Rio de Janeiro, Brasil e atualmente praticado em todo o país, sendo mundialmente conhecido como dança típica brasileira ${ }^{21}$. A música de ritmo empolgante possui diferentes manifestações, como a sofisticada bossa nova, o samba de raiz e as populares marchas de carnaval ${ }^{21}$. Essa mistura de dança e música proporciona uma movimentação espontânea de todo o corpo, o que combina com o espírito alegre e festivo da população brasileira.

Diante do exposto, torna-se plausível a expectativa da influência positiva do samba na saúde geral, com possibilidade de que seja adotado na reabilitação cardíaca, ou, de forma mais abrangente, na reabilitação cardiopulmonar e metabólica (RCPM). O objetivo do estudo foi desenvolver e avaliar um protocolo de samba a ser adotado no treinamento físico de cardiopatas participantes de programa de reabilitação cardíaca.

\section{MATERIAIS E MÉTODO}

Trata-se de estudo longitudinal com amostragem não probabilística. Realizado no Centro de Ciências da Saúde e do Esporte da Universidade do Estado de Santa Catarina SC, Brasil. A pesquisa foi aprovada pelo Comitê de Ética em Pesquisa da Instituição sob o parecer no 109.433. Todos os participantes assinaram Termo de Consentimento Livre e Esclarecido.

Dezenove pacientes foram recrutados em um centro de reabilitação cardíaca da cidade de Florianópolis, SC, Brasil, no período de 4 de fevereiro a 12 de julho de 2013.

Os critérios de inclusão foram: doença arterial coronariana (DAC), estabilidade clínica e sedentarismo há pelo menos três meses e os critérios de exclusão: angina instável, arritmias descompensadas, uso de marca-passo artificial cardíaco, doença pulmonar ou neurológica grave, alterações musculoesqueléticas que limitassem a prática de exercício, alteração do tratamento farmacológico durante o período do estudo e o não cumprimento de pelo menos $80 \%$ das sessões. Quinze sujeitos completaram com êxito todas as etapas do estudo. As características da população são demonstradas na tabela 1.

Tabela 1. Características clínicas da população estudada.

\begin{tabular}{c|c|c}
\hline Variável & Média & DP \\
\hline Idade & 60,74 & 5,96 \\
\hline Sexo & N & $\%$ \\
\hline Homens & & \\
\hline Mulheres & 8 & 53,3 \\
\hline IMC* & 7 & 46,7 \\
\hline Baixo peso & & \\
\hline Normal & 3 & 20 \\
\hline Obeso & 5 & 33,3 \\
\hline Comorbidades & 7 & 46,7 \\
\hline Hipertensão arterial sistêmica & & \\
\hline Diabetes & 12 & 80 \\
\hline IM & 9 & 60 \\
\hline Dislipidemia & 4 & 26,6 \\
\hline Medicamentos & 2 & 13,3 \\
\hline Antiplaquetário & & \\
\hline Betabloqueador & 12 & 80 \\
\hline Diurético & 11 & 73,3 \\
\hline Estatina & 5 & 33,3 \\
\hline Inibidor da ECA & 11 & 73,3 \\
\hline Antagonista receptor de angiotensina & 5 & 33,3 \\
\hline Nitrato & 5 & 33,3 \\
\hline Antiangina & 9 & 60 \\
\hline
\end{tabular}

DP: desvio padrão; N: número da amostra; \%: valor em percentual; IMC: Índice de Massa Corporal; IM: infarto do miocárdio; ${ }^{\prime}$ OMS: Organização Mundial da Saúde, $2000^{22}$ e OPAS: Organização Pan-Americana de Saúde, $2001{ }^{23}$.

\section{Teste Cardiopulmonar}

Os pacientes foram avaliados por meio de teste cardiopulmonar máximo em esteira rolante motorizada (Centurion 200 fabricado pela Micromed Brasília, Brasil), com protocolo em rampa. A troca gasosa foi determinada mediante sistema computadorizado de espirometria e análise de gases, de circuito aberto (Metalyser 3B, Córtex Biophisik, Leipzig - Alemanha) conectado ao software Ergo PC Elite (Micromed ${ }^{\circledR}$, Brasilia, Brasil). Foram determinados os limiares ventilatórios anaeróbio (LV1) e ponto de compensação respiratória (LV2), que serviram de parâmetro para a prescrição da zona ideal de exercício (zona alvo) ${ }^{24}$.

\section{Protocolo de Samba Brasileiro}

As músicas foram previamente selecionadas e classificadas em três andamentos musicais (lento, moderado e rápido) de acordo com suas batidas por minuto (bpm) determinadas por metrônomo da marca Wittner, com andamento lento variando de 40bpm a 72bpm, o médio de $72 \mathrm{bpm}$ a $120 \mathrm{bpm}$ e o rápido de $120 \mathrm{bpm}$ a $208 \mathrm{bpm}^{25}$.

As sessões de samba foram realizadas durante três semanas, com frequência de três vezes por semana, sempre sob orientação de uma 
professora de dança de salão, e supervisão da equipe responsável pelo programa de reabilitação cardíaca.

O estudo foi realizado em duas fases distintas. Na primeira fase foi verificada a adaptação dos pacientes aos passos de samba, durante a realização dos passos nos diferentes andamentos musicais. Em seguida foi verificada a intensidade relativa de treinamento em cada andamento.

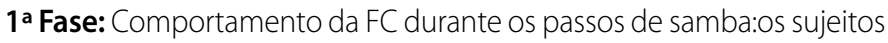
foram divididos de forma aleatória, com sorteio prévio, em três grupos, sendo que o Grupo 1 iniciou as sessões com a utilização do andamento lento, o Grupo 2 com médio e o Grupo 3 com rápido (figura 1).

Em seguida foram selecionados 20 passos de samba (tabela 2), que foram dançados individualmente, durante seis sessões, com diferentes andamentos. No andamento lento, foi utilizada a música "Cajueiro VeIho" (65 bpm) versão cantada por Alcione; para o andamento médio, a música "Samba a Dois", (74 bpm) versão cantada pela banda Los Hermanos; e para o andamento rápido, a música "Curtição №2" do Batucada (124 bpm). Cada passo foi executado pelo tempo de um minuto, nos três diferentes andamentos(lento, médio, rápido), com intervalos entre as execuções. A frequência cardíaca (FC) foi monitorada por cardio-frequencímetros (Marca Polar, modelo RS800cx, da Finlândia).

$2^{2}$ Fase: Intensidade de Treinamento: foram selecionados os passos que foram mais efetivos em manter o percentual da FC entre $60-90 \%$ da $\mathrm{FC}_{\text {pico }}{ }^{26}$. Em seguida foram realizadas três sessões de aproximadamente 40 min. cada uma, com 30 min. destinados ao treinamento principal, $5 \mathrm{~min}$. de aquecimento e $5 \mathrm{~min}$. de volta à calma.

A primeira sessão foi utilizada para familiarização com o treinamento. Na segunda sessão foram selecionadas duas músicas para cada andamento musical. Para a terceira sessão, foi selecionada uma música para o andamento lento, três músicas para o andamento médio e três para o rápido (tabela 3).

Durante as sessões, os sujeitos foram motivados a manter-se a maior parte do tempo na zona alvo ideal de treinamento, entre o primeiro e o segundo limiares ventilatórios, sendo monitorados por cardio-frequencímetros da marca Polar (modelo RS800cx@ da Finlândia) . Durante cada sessão, a percepção de esforço foi registrada em intervalos de 5 min. por meio da escala subjetiva de percepção de esforço de BORG CR-1037 adaptada para uso no Brasil27.

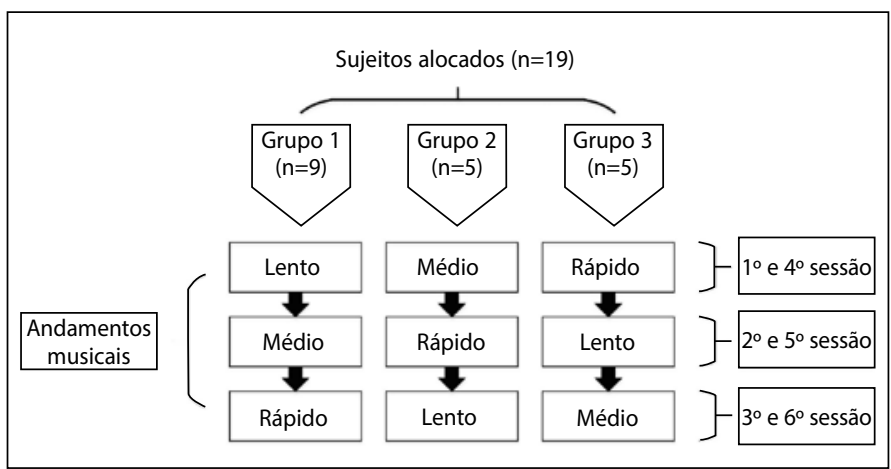

Figura 1. Organograma de aleatorização dos pacientes para execução dos passos.

\section{Análise Estatística}

Os dados foram analisados no software estatístico StatisticalPackage for the Social Sciences (SPSS, versão 20.0) para Windows, com um nível de significância de 5\% para todas as análises. Foi empregada a estatística descritiva, com medidas de porcentagem, tendência central e variabilidade (média e desvio padrão), para a caracterização dos participantes do estudo. Na primeira fase do estudo, foi realizada a moda para verificar a frequência cardíaca que os sujeitos mantiveram por mais tempo, em cada passo, sendo a média utilizada na análise dos grupos. Na segunda fase, para comparação entre as sessões foi utilizado teste $t$ pareado ou Teste de Wilcoxon, conforme a normalidade dos dados.
Tabela 2. Passos de samba inicialmente selecionados para o protocolo.

\begin{tabular}{|c|c|c|}
\hline No & Passos do Samba & Descrição \\
\hline 1 & Transferência de peso & $\begin{array}{c}\text { Pisa no lugar ( } 2 x) \text { alternando direita e esquerda - } \\
\text { Transferência de peso em tempo }\end{array}$ \\
\hline 2 & $\begin{array}{l}\text { Marcação em } \\
\text { três tempos }\end{array}$ & $\begin{array}{c}\text { Pisa no lugar }(3 x) \text { alternando direita e esquerda - } \\
\text { Transferência de peso em contratempo }\end{array}$ \\
\hline 3 & Caminhada & $\begin{array}{l}\text { Caminhada pra frente e voltando para trás - } \\
\text { Transferência de peso em tempo }\end{array}$ \\
\hline 4 & Lado e frente & $\begin{array}{c}\text { Cruza pisando à frente e volta ao lado- } \\
\text { Transferência de peso tempo }\end{array}$ \\
\hline 5 & $\begin{array}{l}\text { Puladinho do Samba } \\
\text { de Gafieira para } \\
\text { frente - No lugar }\end{array}$ & $\begin{array}{c}\text { Abre ao lado e cruza à frente alternando perna } \\
\text { direita e esquerda - } \\
\text { Transferência de peso em contratempo }\end{array}$ \\
\hline 6 & Balanço & $\begin{array}{c}\text { Balancinho para um lado e para o outro - } \\
\text { Transferência de peso em tempo }\end{array}$ \\
\hline 7 & Balanço pra frente & $\begin{array}{l}\text { Balancinho para frente e para trás - } \\
\text { Transferência de peso em tempo }\end{array}$ \\
\hline 8 & $\begin{array}{c}\text { Olha o passarinho ou } \\
\text { passeio do Samba } \\
\text { de Gafieira }\end{array}$ & $\begin{array}{c}\text { Balancinho girando para um lado e voltando a } \\
\text { frente novamente - } \\
\text { Transferência de peso em tempo }\end{array}$ \\
\hline 9 & $\begin{array}{l}\text { Dois pra lá e dois } \\
\text { pra cá }\end{array}$ & $\begin{array}{c}\text { Dois passos para um lado e volta dando dois } \\
\text { passos para o outro - } \\
\text { Transferência de peso em contratempo }\end{array}$ \\
\hline 10 & $\begin{array}{l}\text { Passo básico do } \\
\text { Samba de Gafieira } \\
\text { lateral }\end{array}$ & $\begin{array}{c}\text { Transferência de peso no lugar alternando perna } \\
\text { direita e esquerda e pisa ao lado - } \\
\text { Transferência de peso em contratempo }\end{array}$ \\
\hline 11 & $\begin{array}{l}\text { Passo básico do } \\
\text { Samba de Gafieira }\end{array}$ & $\begin{array}{c}\text { Transferência de peso no lugar e pisa a frente com } \\
\text { a perna direita, Transferência de peso no lugar e } \\
\text { pisa atrás com a perna esquerda - Transferência de } \\
\text { peso em contratempo } \\
\end{array}$ \\
\hline 12 & $\begin{array}{l}\text { Cruzando do Samba } \\
\text { de Gafieira pela } \\
\text { frente }\end{array}$ & $\begin{array}{c}\text { Abre ao lado e cruza a perna pela frente } \\
\text { alternando perna direita e esquerda - Transferência } \\
\text { de peso em contratempo }\end{array}$ \\
\hline 13 & $\begin{array}{l}\text { Cruzando do Samba } \\
\text { de Gafieira por atrás }\end{array}$ & $\begin{array}{c}\text { Abre ao lado e cruza a perna por atrás alternando } \\
\text { perna direita e esquerda - Transferência de peso } \\
\text { em contratempo }\end{array}$ \\
\hline 14 & $\begin{array}{l}\text { Atrás, Lado, Frente } \\
\text { e Cruza }\end{array}$ & $\begin{array}{c}\text { Cruza uma perna à frente em seguida dê um } \\
\text { passo atrás e outro ao lado e cruza novamente - } \\
\text { Transferência de peso em tempo }\end{array}$ \\
\hline 15 & $\begin{array}{l}\text { Atrás, Lado, Frente } \\
\text { e Cruza } 2 x\end{array}$ & $\begin{array}{l}\text { Cruza a mesma perna duas vezes à frente em } \\
\text { seguida dê um passo atrás e outro ao lado e cruza } \\
\text { novamente - Transferência de peso em tempo }\end{array}$ \\
\hline 16 & Caminhada cruzando & $\begin{array}{l}\text { Caminhada cruzando a perna à frente e voltando } \\
\text { cruzando a perna atrás, alternando perna direita e } \\
\text { esquerda - Transferência de peso em tempo }\end{array}$ \\
\hline 17 & Amassa - Cacau & $\begin{array}{l}\text { Pivôs marcando em três tempos, alternando } \\
\text { direita e esquerda e deslocando para frente - } \\
\text { Transferência de peso em contratempo }\end{array}$ \\
\hline 18 & $\begin{array}{l}\text { Puladinho do Samba } \\
\text { de Gafieira para atrás } \\
\text { - Deslocando }\end{array}$ & $\begin{array}{c}\text { Pisa no lugar atrás, marca o pé da frente e pisa } \\
\text { deslocando a perna atrás, alternando perna } \\
\text { direita e esquerda - Transferência de peso } \\
\text { em contratempo. }\end{array}$ \\
\hline 19 & $\begin{array}{l}\text { Samba no pé } \\
\text { no lugar. }\end{array}$ & $\begin{array}{l}\text { Pisa atrás no lugar, marca à frente e atrás, } \\
\text { liberando a outra perna da frente para pisar a atrás } \\
\text { novamente, alternando direita e esquerda - } \\
\text { Transferência de peso em contratempo. }\end{array}$ \\
\hline 20 & $\begin{array}{l}\text { Samba no pé } \\
\text { para trás }\end{array}$ & $\begin{array}{l}\text { Pisa atrás deslocando, marca à frente e atrás, } \\
\text { liberando a outra perna da frente para pisar atrás } \\
\text { novamente, alternando direita e esquerda - } \\
\text { Transferência de peso em contratempo. }\end{array}$ \\
\hline
\end{tabular}

\section{RESULTADOS}

Os resultados das variáveis do teste cardiopulmonar máximo são demonstrados em valores médios da FC do grupo, sendo 104,60 \pm 4,04 bpm no LV1 e 113,26 $\pm 4,43 \mathrm{bpm}$ no LV2. A média da $F C_{\text {pico }}$ foi de $124,73 \pm 4,55$, e do volume do consumo de oxigênio pico (VO2pico) de $18,22 \pm 1,09 \mathrm{ml} / \mathrm{Kg} / \mathrm{min}$.

1a FASE: Comportamento da FC durante os passos de samba: o s valores médios do percentual da FCpico nos 20 passos realizados demonstram que os pacientes se mantiveram dentro da zona de FC de treinamento preconizada (tabela 4).

2a FASE: Intensidade de Treinamento: A tabela 5 demonstra os passos utilizados na aplicação do protocolo, sendo priorizados de acordo com a melhor adaptação dos pacientes. 
Tabela 3. Músicas utilizadas nas sessões de dança 2 e 3 para avaliação do protocolo

\begin{tabular}{|c|c|c|c|c|}
\hline \multirow{3}{*}{ 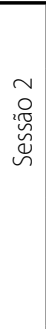 } & \multirow{6}{*}{ 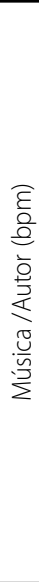 } & $\begin{array}{c}\text { Andamento musical } \\
\text { Lento }\end{array}$ & \begin{tabular}{|c|}
$\begin{array}{c}\text { Andamento musical } \\
\text { Médio }\end{array}$ \\
\end{tabular} & $\begin{array}{c}\text { Andamento musical } \\
\text { Rápido }\end{array}$ \\
\hline & & $\begin{array}{l}\text { Lá Vem Você / } \\
\text { Alcione (72bpm) }\end{array}$ & \begin{tabular}{|} 
Não deixe o samba \\
morrer / Versão \\
cantada por \\
Maria Rita (76 bpm) \\
\end{tabular} & $\begin{array}{c}\text { Samba-Enredo } 1993 \\
\text { Salgueiro / Demá Chagas } \\
\text { (134 bpm) }\end{array}$ \\
\hline & & $\begin{array}{c}\text { Cabide / Marti'nalia } \\
\text { (72bpm) }\end{array}$ & $\begin{array}{l}\text { Don't Worry Be } \\
\text { Happy / Mart'nália } \\
\text { (80 bpm) }\end{array}$ & $\begin{array}{c}\text { Samba-Enredo } 2010 \\
\text { Unidos da Tijuca / } \\
\text { Julio Alves } \\
\text { (138 bpm) }\end{array}$ \\
\hline \multirow{3}{*}{ 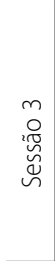 } & & \multirow{3}{*}{$\begin{array}{c}\text { Cabide / Marti'nalia } \\
\text { (72bpm) }\end{array}$} & $\begin{array}{c}\text { Não deixe o samba } \\
\text { morrer / Maria Rita } \\
\text { (76bpm) }\end{array}$ & $\begin{array}{c}\text { Samba-Enredo } 1993 \\
\text { Salgueiro / Demá Chagas } \\
\text { (134bpm) }\end{array}$ \\
\hline & & & $\begin{array}{l}\text { Don't Worry Be } \\
\text { Happy / Mart'nália } \\
\text { (80bpm) }\end{array}$ & $\begin{array}{c}\text { Samba-Enredo } 2010 \\
\text { Unidos da Tijuca / } \\
\text { Julio Alves (138bpm) }\end{array}$ \\
\hline & & & $\begin{array}{c}\text { Flor de Liz / Alcione } \\
\text { (92bpm) }\end{array}$ & $\begin{array}{c}\text { Vou Festejar / } \\
\text { Beth Carvalho (146bpm) }\end{array}$ \\
\hline
\end{tabular}

bpm: batidas por minuto.

Tabela 4. Resposta cronotrópica observada durante a execução dos passos de samba nos diferentes andamentos musicais.

\begin{tabular}{c|c|c|c}
\hline & Andamento lento & Andamento médio & Andamento rápido \\
\hline Passos & ${\text { Média } \% F C_{\text {pico }}}$ & Média $\%$ FC $_{\text {pico }}$ & ${\text { Média } \% \mathrm{FC}_{\text {pico }}}$ \\
\hline 1 & $63,17 \pm 11,35$ & $65,62 \pm 12,40$ & $71,53 \pm 15,27$ \\
\hline 3 & $62,69 \pm 10,97$ & $65,69 \pm 11,81$ & $69,27 \pm 10,25$ \\
\hline 4 & $63,37 \pm 9,63$ & $64,66 \pm 12,21$ & $71,44 \pm 10,42$ \\
\hline 5 & $63,82 \pm 9,16$ & $65,59 \pm 11,52$ & $68,71 \pm 12,85$ \\
\hline 6 & $63,56 \pm 10,73$ & $65,32 \pm 11,52$ & $67,89 \pm 11,03$ \\
\hline 7 & $62,13 \pm 10,69$ & $66,40 \pm 14,13$ & $74,59 \pm 12,39$ \\
\hline 8 & $64,36 \pm 15,87$ & $65,51 \pm 12,07$ & $74,21 \pm 13,99$ \\
\hline 9 & $62,14 \pm 10,38$ & $64,75 \pm 10,38$ & $69,16 \pm 10,34$ \\
\hline 10 & $62,41 \pm 10,12$ & $65,25 \pm 11,06$ & $69,58 \pm 11,08$ \\
\hline 11 & $61,91 \pm 10,62$ & $64,51 \pm 12,58$ & $70,87 \pm 10,54$ \\
\hline 12 & $62,37 \pm 12,68$ & $66,92 \pm 11,64$ & $69,36 \pm 11,55$ \\
\hline 13 & $63,79 \pm 11,05$ & $64,90 \pm 11,89$ & $70,05 \pm 69,58$ \\
\hline 14 & $66,13 \pm 17,88$ & $63,94 \pm 12,19$ & $69,07 \pm 11,22$ \\
\hline 15 & $62,77 \pm 10,28$ & $63,56 \pm 12,20$ & $70,68 \pm 11,19$ \\
\hline 16 & $63,26 \pm 10,65$ & $64,13 \pm 10,22$ & $71,53 \pm 12,06$ \\
\hline 17 & $64,43 \pm 11,32$ & $65,30 \pm 13,92$ & $71,37 \pm 13,54$ \\
\hline 18 & $63,13 \pm 8,11$ & $64,69 \pm 12,11$ & $69,39 \pm 11,75$ \\
\hline 19 & $63,53 \pm 10,58$ & $64,24 \pm 12,22$ & $66,35 \pm 12,23$ \\
\hline 20 & $64,90 \pm 11,02$ & $68,25 \pm 13,08$ & $69,63 \pm 10,84$ \\
\hline Total & $60,34 \pm 14,18$ & $69,36 \pm 12,18$ & $73,76 \pm 13,15$ \\
\hline Percentual da frequência cardíaca pico. & $65,42 \pm 1,41$ & $70,42 \pm 2,07$ \\
\hline 16 & & & \\
\hline
\end{tabular}

Tabela 5. Passos de samba definitivamente integrados ao protocolo, conforme adaptação dos pacientes.

\begin{tabular}{c|c}
\hline \multicolumn{2}{c}{ Passos de melhor adaptação } \\
\hline 1 & Transferência de peso \\
\hline 2 & Marcação em três tempos \\
\hline 3 & Caminhada \\
\hline 4 & Lado e frente \\
\hline 5 & Puladinho do Samba de Gafieira para frente - No lugar \\
\hline 6 & Balanço pra frente \\
\hline 7 & Olha o passarinho ou passeio do Samba de Gafieira \\
\hline 8 & Dois pra lá e dois pra cá \\
\hline 9 & Passo básico do Samba de Gafieira lateral do Samba de Gafieira \\
\hline 10 & Cruzando do Samba de Gafieira pela frente \\
\hline 12 & Atrás, Lado, Frente e Cruza \\
\hline 14 & Atrás, Lado, Frente e Cruza 2x \\
\hline 15 & Caminhada cruzando \\
\hline 16 & Samba no pé no lugar. \\
\hline 19 & Samba no pé para trás \\
\hline 20 & Para \\
\hline
\end{tabular}

Foram excluídos do protocolo 3 passos: 13 Cruzando do Samba de Gafieira por trás; 17 Amassa-Cacau e 18 Puladinho do Samba de Gafieira para trás - Deslocando.
Durante as sessões de treinamento os sujeitos permaneceram $86,57 \pm 4,17 \%$ do tempo total das sessões na zona de treinamento proposta para treinamento físico em reabilitação que compreende os valores entre $60 \%$ e $90 \%$ da $\mathrm{FC}_{\text {pico }}$ avaliado pelo teste cardiopulmonar. Quando analisado o tempo total em que os indivíduos permaneceram na zona-alvo considerada ideal de treinamento para esta população, em que a FC deve se situar entre o primeiro e o segundo limiar ventilatório, este valor ficou em $76,79 \pm 2,28 \%$ do tempo, sendo $75,18 \pm 16,03 \%$ do

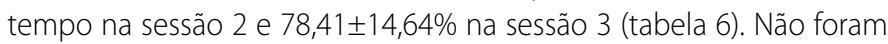
encontradas diferenças estatisticamente significativas entre as sessões 2 e 3, nas variáveis estudadas. Os resultados são independentes do tempo disposto para cada andamento, percebendo-se que há uma tendência a maior permanência em zona alvo quando otimizado o tempo nos andamentos médio e rápido. Quando analisados os andamentos musicais de forma independente, verificamos que os sujeitos permaneceram dentro da zona alvo de treinamento, em maior parte do tempo no andamento rápido, segundamente, no andamento médio e por último no andamento lento, não sendo verificadas diferenças significativas entre as duas sessões propostas.

A figura 2 demonstra os valores médios da escala de percepção subjetiva de esforço modificada - BORG, em intervalos de 5 minutos, nas sessões 2 e 3 . Pode-se observar que, em ambas as aulas, os valores permaneceram entre $2,07 \pm 1,18$ e 3,53 $\pm 2,25$, correspondentes às percepções de esforço leve e moderada, sem diferenças estatisticamente significativas.

\section{DISCUSSÃO}

Na literatura atual é limitado o número de estudos que investigam os efeitos da dança como forma alternativa na reabilitação para cardiopatas ${ }^{11-13,18}$. Não foi encontrado na literatura um protocolo de dança que seja reprodutível para uso em programas de reabilitação. Tendo em vista os resultados relacionados à resposta cronotrópica e percepção de esforço, o protocolo de samba brasileiro pode ser uma alternativa eficaz de treinamento para pacientes com doença arterial coronariana. O American Collegeof Sport Medicine ${ }^{26}$ e a Sociedade Brasileira de Cardiologia ${ }^{4}$ recomendam para coronariopatas a prática regular de exercício físico com intensidade entre $60 \%$ a $90 \%$ da $\mathrm{FC}_{\text {pico, }}$ com percepção de esforço em nível moderado. Nosso estudo demonstrou que durante a primeira fase, com todos os passos de samba, nos três andamentos musicais, houve uma resposta cronotrópica dentro da faixa preconizada.

Recomenda-se como forma ideal de prescrição de treinamento para cardiopatas a zona alvo compreendida entre o primeiro e o segundo limiar ventilatório do teste ergoespirométrico ${ }^{4,26,28}$. O nosso protocolo de samba brasileiro manteve os pacientes em torno de $77 \%$ do tempo de atividade com FC dentro da zona alvo ideal de treinamento, com percepção de esforço subjetiva de leve a moderada. O nível de percepção de esforço encontrado pode ser justificado pelo fato de que exercícios realizados com a adição de música são capazes de ativar áreas cerebrais específicas, que estimulam sentimentos de prazer e euforia e desviam a percepção humana de seu próprio esforço29,30.

Sabe-se que dentro de cada andamento musical há variações de bpm ${ }^{25}$, desta forma, na prescrição do protocolo a escolha do bpm da musica é algo relevante, pois a proximidade ao limite superior ou inferior poderá proporcionar diferentes respostas cardiovasculares, servindo como alternativa para evolução do treinamento.

Os três diferentes andamentos musicais devem ser abordados durante as sessões, utilizados de forma gradativa, iniciando sempre do andamento lento, seguido do médio e rápido, para maior adaptação cardiovascular. Os dados apresentados neste estudo demonstraram que o andamento musical lento pode ser utilizado como aquecimento e desaquecimento para coronariopatas com melhor aptidão cardiopulmonar, ou como 
Tabela 6. Permanência na zona alvo (em relação $\mathrm{aFC}_{\text {pico }}$ ) e zona alvo ideal de treinamento (entre limiares ventilatórios) nas sessões 2 e 3.

\begin{tabular}{|c|c|c|c|c|c|c|c|c|}
\hline Andamento Musical & & $2(\%)$ & & $3(\%)$ & Média Total por & amento musical (\%) & & \\
\hline & Zona alvo* & Zona alvo ideal $^{\dagger}$ & Zona alvo* & Zona alvo ideal $^{\dagger}$ & Zona alvo* & Zona alvo ideal $^{\dagger}$ & $\mathrm{p}^{ \pm}$ & $p^{\S}$ \\
\hline Médio & $87,40 \pm 27,81$ & $18,52 \% \pm 36,04$ & $87,77 \pm 29,85$ & $20,00 \pm 30,50$ & $87,59 \% \pm 0,26$ & $19,25 \% \pm 1,04$ & 1,00 & 0,735 \\
\hline Total & $84,69 \pm 29,95$ & $75,18 \% \pm 16,03$ & $89,08 \pm 26,60$ & $78,41 \% \pm 14,64$ & $86,57 \% \pm 4,17$ & $76,79 \% \pm 2,28$ & 0,273 & 0,790 \\
\hline
\end{tabular}

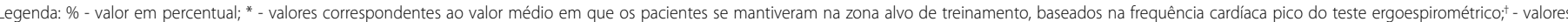

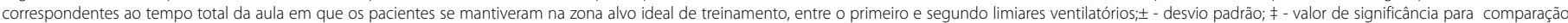

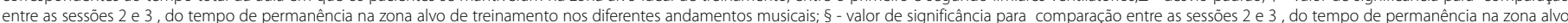
ideal de treinamento nos diferentes andamentos musicais.

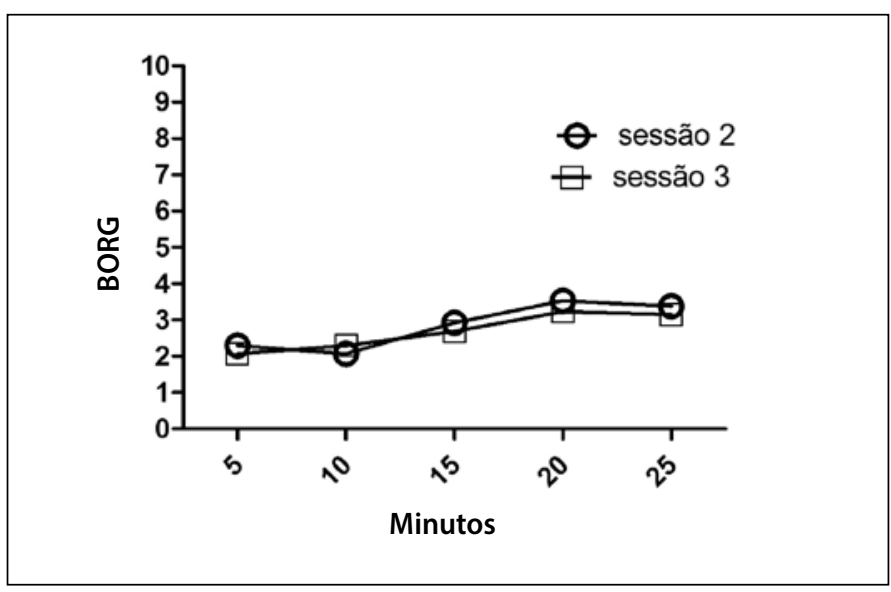

Figura 2. Percepção subjetiva de esforço modificada nas sessões 2 e 3 do protocolo.

fase principal para os pacientes menos condicionados. Os andamentos médio e rápido, por proporcionarem maior resposta cardiovascular no treinamento, devem ser utilizados durante a parte principal das aulas dos pacientes com status funcional elevado.

Diante do exposto, este estudo apresentou informações originais no processo de construção e aplicação de um protocolo de dança

\section{REFERÊNCIAS}

1. Perk J, Backer GD, Gohlke H, Graham I, Reiner Z, Verschuren WMM, et al. European Guidelines on cardiovascular disease prevention in clinical practice (version 2012). Eur Heart J. 2012; 33:1635-701.

2. Moraes RS, Nóbrega $A C L$, Castro RRT, Negrão CE, Stein R, Serra SM, et al. Diretriz de Reabilitação Cardíaca. Arq. Bras. Cardiol. 2005;84(5):431-40.

3. Leon AS, Chair MS, Franklin BA, Costa F, Balady GJ, Berra KA, et.al. Cardiac Rehabilitation and Secondary Prevention of Coronary Heart Disease: An American Heart Association Scientific Statement From the Council on Clinical Cardiology (Subcommittee on Exercise, Cardiac Rehabilitation, and Prevention) and the Council on Nutrition, Physical Activity, and Metabolism (Subcommittee on Physical Activity), in Collaboration with the American Association of Cardiovascular and Pulmonary Rehabilitation. 2005;111(3): 369-76.

4. Ghorayeb N, Costa RVC, Castro I, Daher DJ, Oliveira Filho JA, Oliveira MAB, et al. Diretriz em Cardiologia do Esporte e do Exercício da Sociedade Brasileira de Cardiologia e da Sociedade Brasileira de Medicina do Esporte. Arq. Bras. Cardiol. 2013;100(1) Supl.2:1-41

5. Heran BS, Chen JM, Ebrahim S, Moxham T, Oldridge N, Rees K, et al. Exercise-based cardiac rehabilitation for coronary heart disease.The Cochrane Database of Systematic Reviews 2011;6(7)

6. Daly J, Sindone AP, Thompson DR, Hancock K, Chang E, Davidson P. Barriers to participation in and adherence to cardiac rehabilitation programs: a critical literature review. ProgCardiovascNurs. 2002;17(1):8-17.

7. Farley RL, Wade TD, Birchmore L. Factors Influencing Attendance at Cardiac Rehabilitation among Coronary Heart Disease Patients. Eur J CardiovascNurs. 2003;2:205.

8. Guimarães GV, Carvalho VO, Bocchi EA, d'Avila VM. Pilates in heart failure patients: a randomized controlled pilot Trial. CardiovascTher. 2012;30:351-6.

9. Piliae-Taylor RE. Tai Chi as an Adjunct to Cardiac Rehabilitation Exercise Training. J CardiopulmRehabil. 2003;23:90-6.

10. Look AM, Kaholokula JK, Carvahio A, Seto TB, Silva M. Developing a culturally based cardiac rehabilitation program: The HELA Study. Prog Community Health Partnersh. 2012;6(1):103-10.

11. Belardinelli R, Lacalaprice F, Ventrella C, Volpe L, Faccenda E. Waltz dancing in patients with chronic heart failure: new form of exercise training. CORC Heart Fail. 2008;(1):107-14.

12. Schenkel IC, Bündchen DC, Quites MP, Santos RZ, Santos MB, Carvalho T. Comportamento da pressão arterial em hipertensos após única sessão de caminhada e de dança de salão: estudo preliminar. RevBrasCardiol. 2011;24(1):26-32

13. Kaltsatou ACH, Kouidi El, Anifanti MA, Douka SI, Deligiannis AP. Functional and psychosocial effects of either a traditional dancing or a formal exercising training program in patients with chronic heart failure: a comparative randomized controlled study. ClinRehabil. 2014;28(2):128-38.

14. Hui E, Chui BT, Woo J. Effects of dance on physical and psychological well-being in older persons. Arch GerontolGeriatr. 2009;49:e45-e50. para participantes de programa de reabilitação cardíaca, que se mostrou capaz de colocar com segurança pacientes com doença arterial coronariana na zona alvo proposta para treinamento físico sem intercorrências médicas.

\section{CONCLUSÃO}

Os pacientes demonstraram boa adaptação ao protocolo de samba brasileiro, que se mostrou efetivo como proposta de treinamento na reabilitação cardíaca, considerando os parâmetros relacionados à frequência cardíaca, limiares ventilatórios e percepção de esforço.

\section{AGRADECIMENTOS}

Os autores agradecem aos integrantes do Núcleo de Cardiologia e Medicina do Exercício (NCME) da Universidade do Estado de Santa Catarina (UDESC) por auxiliarem na execução da coleta de dados. A todos os pacientes que participaram das coletas durante a pesquisa e aos pesquisadores colaboradores Mirele Porto Quites, Vitor Angarten, e o bailarino Ricardo Koscialkowski Tetzner, contribuindo no planejamento da pesquisa e auxiliando na melhor descrição dos movimentos do samba.

Todos os autores declararam não haver qualquer potencial conflito de interesses referente a este artigo.

15. Kirsch $L P$, Drommelschmidt KA, Cross ES. The impact of sensorimotor experience on affective evaluation of dance. FronHumNeurosci. 2013, 7(521):1-10.

16. Bradt J, Goodill SW, Dileo C. Dance/movement therapy for improving psychological and physical outcomes in cancer patients. The Cochrane Database of Systematic Reviews 2011;5(10).

17. Foster ER, Golden L, Duncan RP, Earhart GM. A community-based Argentine tango dance program is associated with increased activity participation among individuals with Parkinson disease. Arch Phys Med Rehabil. 2013;94(2):240-9.

18. Aweto HA, Owoeye OB, Akinbo SR, Onabajo AA. Effects of dance movement therapy on selected cardiovascular parameters and estimated maximum exygen consumption in hypertensive patients. Nig Q J Hosp Me. 2012;22(2):125-9.

19. Karageorghis Cl, Jones L, Priest DL, Akers Rl, Clarke A, Perry JM, et al. Revisiting the relationship between exercise heart rate music tempo preference. Res Q Exerc Sport. 2011;82(2):274-84.

20. Birnbaum L, Boone T, Huschle B. Cardiovascular Responses To Music Tempo During Steady-State Exercise. J ExercPhysiol 2009;12(1):50-6.

21. Perna MA. Samba de Gafieira - a História da Dança de Salão Brasileira. Rio de Janeiro: s/ editora; 2001.

22. World Health Organization (WHO). The problem of overweight and obesity. In: Obesity: preventing and managing the global epidemic. Geneva; 2000. (Technical Report Series).

23. Organização Pan-americana de Saúde (OPAS). XXXVI Reunión Del Comité Asesor de Investigaciones em Salud - EncuestraMulticêntrica - Salud Beinestar y Envejecimento (SABE) enAmerica Latina y el Caribe - Informe Preliminar. 2001. [Acesso em 2013 dez 06]. Disponível em: http://www.paho.org/ Spanish/HDP/HDR/CAIS-01-05.PDF.

24. Meneghelo RS, Araújo CGS, Stein R, Mastrocolla LE, Albuquerque PF, Serra SM et al. Sociedade Brasileira de Cardiologia. III Diretriz da Sociedade Brasileira de Cardiologia sobre Teste Ergométrico. ArqBrasCardiol. 2010;95(5 supl.1):1-26

25. Bohumil MED. Teoria da Musica. 3a Edição. Musimed; 1996.

26. American College of Sports Medicine Position Stand: Exercise For Patients With Coronary Artery Disease. Med. Sci. Spans. Exenz. 1994;26( 3):i-v.

27. Borg G. Escalas de Borg para a Dor e Esforço Percebido. São Paulo: Manole, 2000.

28. Godoy M, Bellini AJ, Passaro LC, Mastrocolla LE. I Consenso nacional de Reabilitação Cardiovascular (Fase Crônica). Arq Bras Cardiol.1997;69 (4):267-91

29. Chanda ML \& Daniel JL. The neurochemistry of music. Trends Cogn Sci. 2013;17(4):179-93.

30. Zatorre RJ, Salimpoor VN. From perception to pleasure: music and its neural substrates. PNAS 2013;110 (suppl. 2):10430-7. 